\title{
DIAGNOSTIC UTILITY OF ADC VALUE OF THE SPLEEN IN ASSESSING SEVERITY OF PORTAL HYPERTENSION IN CIRRHOSIS
}

\author{
Komalavalli Subbiah' ${ }^{1}$, Balaji Ayyamperumal2 , Amarnath Chellathurai ${ }^{3}$ Sathyan Gnanasigamani4 ${ }^{4}$ Suhashini Balasubramaniam ${ }^{5}$, \\ Sivakumar Kannappan ${ }^{6}$
}

\begin{abstract}
${ }^{1}$ Assistant Professor, Department of Radiodiagnosis, Stanley Medical College, Chennai, Tamilnadu, India. ${ }^{2}$ Assistant Professor, Department of Radiodiagnosis, Stanley Medical College, Chennai, Tamilnadu, India. 3 Professor, Department of Radiodiagnosis, Stanley Medical College, Chennai, Tamilnadu, India. ${ }^{4}$ Associate Professor, Department of Radiodiagnosis, Stanley Medical College, Chennai, Tamilnadu, India. ${ }_{5}^{5}$ Associate Professor, Department of Radiodiagnosis, Stanley Medical College, Chennai, Tamilnadu, India. ${ }^{6}$ Assistant Professor, Department of Radiodiagnosis, Stanley Medical College, Chennai, Tamilnadu, India.
\end{abstract}

\section{ABSTRACT}

\section{BACKGROUND}

ADC (Apparent diffusion coefficient) value of the diffusion weighted imaging is a measure of the haemodynamics of the organ. Portal hypertension (PH) is one of the essential manifestations of chronic liver disease. ADC was used in previous studies to indirectly assess fibrosis of the liver. However, no studies have evaluated the ADC value of the spleen to assess the PH features in cirrhosis.

Aims and Objectives- We did the study to analyse the correlation of ADC value of the spleen in cirrhotic patients, to the severity of $\mathrm{PH}$ in the form of clinically significant $\mathrm{PH}(\mathrm{CSPH})$ and also the surrogate markers of $\mathrm{PH}$.

\section{MATERIALS AND METHODS}

We prospectively evaluated patients with chronic liver disease in our hospital from the month of June 2017 to October 2017 with MRI of the abdomen. 1.5-tesla diffusion-weighted imaging with ADC mapping was performed with $b$ value of 0,300 and 500 and the ADC value of the spleen and liver were calculated. They were correlated with the severity of the PH in terms of CSPH and PH surrogate markers.

\section{RESULTS}

In chronic liver disease/ cirrhotic patients, we found a significant correlation between ADC value of the spleen and CSPH as well as PH surrogate markers in the form of grade of oesophageal varices and symptomatic hypersplenism.

\section{CONCLUSION}

Our study showed that ADC value of the spleen correlated well with the severity of PH. ADC measurements may allow for noninvasive evaluation of portal pressure and even in assessment of treatment response.

\section{KEY WORDS}

Diffusion Magnetic Resonance Imaging, Liver Cirrhosis, Hypertension, Portal.

HOW TO CITE THIS ARTICLE: Subbiah K, Ayyamperumal B, Chellathurai A, et al. Diagnostic utility of ADC value of the spleen in assessing severity of portal hypertension in cirrhosis. J. Evolution Med. Dent. Sci. 2018;7(23):2814-2818, DOI: $10.14260 /$ jemds/2018/635

\section{BACKGROUND}

Chronic liver disease is a major spectrum of disease due to various aetiologies and portal hypertension $(\mathrm{PH})$ is an essential manifestation of end-stage liver disease. However, accurate evaluation of $\mathrm{PH}$ severity and its management depends on invasive investigations like repeated upper gastrointestinal endoscopy and hepatic venous wedge pressure monitoring. Non-invasive investigations like ultrasound (US) abdomen and Doppler, though simple, repeatable and cost effective are still plagued by its observer variations due to non-standardised techniques and inferior efficacy of cancer surveillance. ${ }^{1,2}$

'Financial or Other Competing Interest': None.

Submission 19-04-2018, Peer Review 20-05-2018,

Acceptance 28-05-2018, Published 04-06-2018.

Corresponding Author:

Balaji Ayyamperumal,

Assistant Professor,

Department of Radiodiagnosis,

Stanley Medical College,

Chennai, Tamilnadu, India.

E-mail: balajianaesthetist@gmail.com

DOI: $10.14260 /$ jemds $/ 2018 / 635$

\section{(c) (1) $(9$}

Recently, magnetic resonance imaging (MRI) has been used increasingly in the evaluation of cirrhotic patients, more for cancer evaluation and surveillance. Diffusion weighted imaging (DWI) and MRI elastography (MRE) were used for assessing the degree of liver fibrosis. ${ }^{3,4}$ However, very few studies have assessed the MRI characteristic of the spleen and its haemodynamics in cirrhosis and portal hypertensive patients.5,6

Apparent diffusion co-efficient (ADC) obtained using DWI is a useful parameter in assessing the relative haemodynamics of any organ. Many series and reports have revealed that liver ADC was significantly reduced in cirrhotic compared to non-cirrhotic patients. ${ }^{7}$ This is most probably related to distortion of lobular architecture of the liver due to fibrosis. Also, these results have shown that ADC values are useful as a predictive marker of the severity of liver fibrosis, especially moderate and advanced stage of fibrosis. In these studies, spleen ADC has been used as a reference organ for standardisation and in one study it correlates with the stage of cirrhosis. However, splanchnic hyperaemia is the main contributor of portal hypertension in advanced cirrhosis and ADC value of the spleen should be a true reflection of the portal hypertension and not the stage of cirrhosis. Portal 
hypertension need not correlate with the stages of cirrhosis. Hence, we hypothesised that the ADC value of the spleen should be a reliable predictor of the severity of portal hypertension and should correlate well with the portal hypertension parameters in the form of grade of varices, gastrointestinal bleed, spleen size, platelet count, hypersplenism etc. 8

The primary objective of this study is to analyse the correlation of ADC value of the spleen in chronic liver disease patients, to the severity of portal hypertension in the form of clinically significant portal hypertension (CSPH) and also the surrogate markers of portal hypertension in the form of grade of oesophageal varices, incidence of gastrointestinal (GI) bleeds, hypersplenism and size of the spleen. We also tried to analyse the correlation of the spleen and liver ADC to the severity of cirrhosis in terms of Child-Turcotte-Pugh's class (CTP) and model for End-Stage Liver Disease (MELD) score. ${ }^{9}$

\section{MATERIALS AND METHODS}

This prospective descriptive study was performed in our hospital from January 2017 to October 2017 on consecutive chronic liver disease patients referred to our Radiology Department after obtaining clearance from our Institutional Ethical Committee. As this study is the first of its kind done on portal hypertension patients, we initially planned to find out any difference and the characteristics of the MRI (DWI) spleen findings of chronic liver disease/ cirrhosis patients compared to a control group with no liver disorders. For control, patients who undergo MRI abdomen investigation for other reasons with normal liver function test were included and were analysed.

Patients admitted with clinical and examination findings of chronic liver disease/ cirrhosis were evaluated with detailed history. Their investigations were reviewed to confirm the aetiology, evidence of hypersplenism (haemoglobin, white blood cell count and platelet count), CTP class and MELD score to assess the severity of chronic liver disease. Upper gastrointestinal endoscopy findings of the patient done at the initial visit or the highest grade of the oesophageal varices were noted along with sonographic findings of the size and echotexture of the liver along with spleen size, portal vein diameter and grade of ascites (no/moderate/severe). Patients were analysed before starting treatment for portal hypertension, as this might be a confounding variable on the ADC value. Finally, the patients were subjected for MRI examination after informed consent. The study was done in a 1.5-tesla MRI scanner (Siemens Magnetom Symphony) with b value of 0, 300 and 500 for DWI. Routine T1W1, T2W1 and in phase/ opposed phase imaging was done as part of protocol. Slice thickness of $5 \mathrm{~mm}$, matrix size of $256 * 256$ were used. ADC value of the spleen along with liver was calculated.

Clinically significant portal hypertension (CSPH) was defined as the presence of either oesophageal or gastric variceal bleed, portal hypertensive gastropathy or thrombocytopaenia (platelet count < 100000/cu.mm) associated with splenomegaly. Oesophageal varices were graded according to Conn's classification. ${ }^{10}$ Hypersplenism was defined as the presence of splenomegaly with a defect in any one of the peripheral cell lines (anaemia- haemoglobin less than $8 \mathrm{gm} / \mathrm{dL}$ with normocytic and normochromic appearance in peripheral smear; a leukocyte count of $<3500 /$ cu.mm and a platelet count of $<150000 /$ cu.mm. ${ }^{11}$ Degree of splenomegaly was classified using Hackett's classification. The severity of hepatic encephalopathy is graded with the "West Haven" criteria. 12

Statistical analysis was done using SPSS ver. 17. Student's ' $t$ ' test was used in the comparison of numerical variables (mean ages, liver size, spleen size, PV diameter, laboratory values, liver and spleen ADC values) between the patient group and the control group. ANOVA was used in comparison of CTP class and MELD score to the ADC value of the patients. Again student's ' $t$ ' test was used in comparison of the ADC value of the patient to different portal hypertension surrogate markers, degree of liver fibrosis and CSPH. Differences were considered to be statistically significant at $\mathrm{p}<0.05$.

\section{RESULTS}

\section{ADC Spleen- Control vs. Chronic Liver Disease Patients}

We initially compared 15 patients from each group (Case and control) to analyse the difference in MRI findings of the spleen in DWI. We analysed the demographics of both groups and found to be matched with no significant difference between the age and sex of the patients (Median age 43 vs. $42, \mathrm{p}=0.9$, sex 12 vs. 15 males, $\mathrm{p}=0.6$ ). When the clinical and biochemical parameters were compared between the two groups, there were obviously significant differences in parameters of chronic liver disease like hypersplenic features, liver span, spleen span and serum bilirubin, serum creatinine and INR.

\section{ADC Characteristics of the Spleen and Liver- Case vs. Control}

When the ADC value $\left(\mathrm{mm}^{2} / \mathrm{s}\right)$ of the spleen and liver were analysed between the case and control group, we found ADC value of the spleen increased $(117.4 \pm 28.4$ vs. $80.7 \pm 9.1$ $\mathrm{p}=0.04)$ significantly in chronic liver disease patients, whereas ADC value of the liver significantly decreased $(107.2$ \pm 41.8 vs. $338.9 \pm 31.1, p=0.001$ ) in the same group, (Table-1).

\section{ADC Spleen and Chronic Liver Disease}

We analysed the 51 chronic liver disease patients of various aetiologies managed in our hospital from January 2017 . October 2017. Out of the 51 chronic liver disease patients, most of them belonged to CTP class A (29/51) status with 9 patients of CTP class $C$ status. Their mean age was $49.6 \pm 12.3$ years with 38 of them males. Most of them had hypersplenism with their mean haemoglobin of $8.42 \pm 2.1$ gms $\%$, mean WBC count of $5820 \pm 1720 /$ cu.mm and mean platelet count of $100313 \pm 25189 /$ cu.mm. Out of the 51 patients, 32 patients were bleeders with 4 patients having grade 4 varices. Their median MELD score was 11.

\footnotetext{
ADC of the Spleen with Severity of Portal Hypertension We analysed the ADC mapping of the spleen for the 51 patients and compared it between the 39 patients who had clinically significant portal hypertension (CSPH) to the group of 12 patients who had no CSPH. We found the ADC value of the spleen was significantly higher $(125.2 \pm 29.6$ vs. $84.1 \pm 1.6$, $\mathrm{p}$ value $=0.05$ ) in the CSPH group; however, the ADC value of the liver is non-significantly lower $(84.1 \pm 1.6$ vs. $125.2 \pm 29.6$,
} 
$\mathrm{p}$ value $=0.09)$ in the CSPH group compared to the non-CSPH group, Table 2 .

\section{ADC of the Spleen and Portal Hypertension Surrogate} Markers

We also analysed the ADC value of the spleen with the different portal hypertension surrogate markers and found that though ADC of the spleen was non-significantly higher in the presence of increased severity of portal hypertension, it is the oesophageal varices > grade 2 and symptomatic hypersplenism which had significantly higher spleen ADC compared to the other group, Table 3.

\section{ADC Spleen and Liver vs. CTP Class Status}

As evaluated in the past studies, we also analysed the ADC value of the spleen and liver for different CTP class patients and found that as the CTP class worsens, the ADC value of the liver decreased $(140.1 \pm 13.4$ vs. $120.2 \pm 11.3$ vs. $82.0 \pm 9.3, p$ value $=0.05)$, which was statistically significant. However, though the ADC of the spleen increased proportionately to the CTP class $(85.9 \pm 9.4$ vs. $101.4 \pm 11.4$ vs. $121.6 \pm 14.2$, pvalue $=0.12$ ), it was not statistically significant, Table 4 .

ADC Spleen and Liver vs. Model for End-Stage Liver Diseases (MELD)

We in addition analysed the ADC value of the spleen and liver to different levels of MELD score and again found that the ADC value of the liver significantly decreased $(135.1 \pm 11.4$ vs. $113.2 \pm 16.3$ vs. $83.9 \pm 10.3$, $p$ value $=0.04$ ) according to the increasing MELD score. Here also the ADC of the spleen was non-significantly increasing $(89.9 \pm 9.4$ vs. $99.4 \pm 11.4$ vs. $127.6 \pm 14.2, \mathrm{p}$ value $=0.09$ ) to the increasing MELD score, Table 5 .

\begin{tabular}{|c|c|c|c|}
\hline Variables & Case (15) & Control (15) & P value \\
\hline ADC Liver $\left(\mathrm{mm}^{2} / \mathrm{s}\right)$ & $107.2 \pm 41.8$ & $338.9 \pm 31.1$ & 0.001 \\
\hline ADC Spleen $\left(\mathrm{mm}^{2} / \mathrm{s}\right)$ & $117.4 \pm 28.4$ & $80.7 \pm 9.1$ & 0.04 \\
\hline Table 1. Mean ADC Spleen and Liver $\left(\mathrm{mm}^{2} / \mathbf{s}\right)$ - Case vs. \\
Control \\
\hline
\end{tabular}

\begin{tabular}{|c|c|c|}
\hline Variables & $\begin{array}{c}\text { ADC Spleen } \\
\left(\mathbf{m m}^{\mathbf{2}} \mathbf{/ s}\right)\end{array}$ & $\begin{array}{c}\text { ADC Liver } \\
\mathbf{( m m}^{\mathbf{2}} \mathbf{\text { s }} \mathbf{~}\end{array}$ \\
\hline CSPH (39) & $125.2 \pm 29.6$ & $89.2 \pm 20.1$ \\
\hline No CSPH (12) & $84.1 \pm 1.6$ & $134.3 \pm 11.4$ \\
\hline P value & $\mathbf{0 . 0 5}$ & 0.09 \\
\hline
\end{tabular}

Table 2. Correlation between Mean ADC value of Spleen and Liver to Clinically Significant Portal Hypertension (CSPH)

\begin{tabular}{|c|c|c|}
\hline $\begin{array}{c}\text { Portal Hypertension } \\
\text { Surrogates }\end{array}$ & ADC Spleen $\left(\mathrm{mm}^{2} / \mathbf{s}\right)$ & P value \\
\hline $\begin{array}{c}\text { Bleeder (32) vs. Non- } \\
\text { bleeder (19) }\end{array}$ & $120.2 \pm 29.6$ vs. $86.1 \pm 1.6$ & 0.06 \\
\hline $\begin{array}{c}\text { Grade of Oesophageal } \\
\text { Varices (<=2 vs. }>2)\end{array}$ & $93.1 \pm 9.9$ vs. $126.6 \pm 20.2$ & 0.04 \\
\hline $\begin{array}{c}\text { Symptomatic } \\
\text { Hypersplenism (19) vs. } \\
\text { Asymptomatic } \\
\text { Hypersplenism (32) }\end{array}$ & $123.3 \pm 23.1$ vs. $90.2 \pm 11.3$ & 0.02 \\
\hline $\begin{array}{c}\text { PV Diameter } \\
(<=13 \text { mm vs. }>13 \text { mm) }\end{array}$ & $112.3 \pm 23.1$ vs. $123.3 \pm 8.9$ & 0.12 \\
\hline $\begin{array}{c}\text { Splenomegaly (<= } 13 \mathrm{~cm} \\
\text { vs. }>13 \mathrm{~cm})\end{array}$ & $109.5 \pm 9.1$ vs. $123.4 \pm 7.8$ & 0.23 \\
\hline $\begin{array}{c}\text { Table 3. Mean ADC of the Spleen and Portal Hypertension } \\
\text { Surrogate Markers }\end{array}$ \\
\hline
\end{tabular}

\begin{tabular}{|c|c|c|}
\hline Child Pugh Class & ADC Liver $\mathbf{~ m m}^{\mathbf{2}}$ /s) & ADC Spleen $\mathbf{~ m m}^{\mathbf{2}} \mathbf{~ s )}$ \\
\hline A & $140.1 \pm 13.4$ & $85.9 \pm 9.4$ \\
\hline B & $120.2 \pm 11.3$ & $101.4 \pm 11.4$ \\
\hline C & $82.0 \pm 9.3$ & $121.6 \pm 14.2$ \\
\hline P value & $\mathbf{0 . 0 5}$ & 0.12 \\
\hline \multicolumn{3}{|c|}{ Table 4. Mean ADC Spleen/Liver vs. Child-Pugh Class } \\
Status \\
\hline
\end{tabular}

\begin{tabular}{|c|c|c|}
\hline MELD & ADC Liver ( $\mathbf{m m}^{\mathbf{2}}$ /s) & ADC Spleen $\left(\mathbf{m m}^{\mathbf{2}} \mathbf{\text { s) }}\right.$ \\
\hline$>15$ & $135.1 \pm 11.4$ & $89.9 \pm 9.4$ \\
\hline $16-25$ & $113.2 \pm 16.3$ & $99.4 \pm 11.4$ \\
\hline$>25$ & $83.9 \pm 10.3$ & $127.6 \pm 14.2$ \\
\hline P value & 0.04 & 0.09 \\
\hline \multicolumn{2}{|c|}{ Table 5. Mean ADC Spleen/Liver vs. Model for End-Stage } \\
Liver Disease (MELD) \\
\hline
\end{tabular}

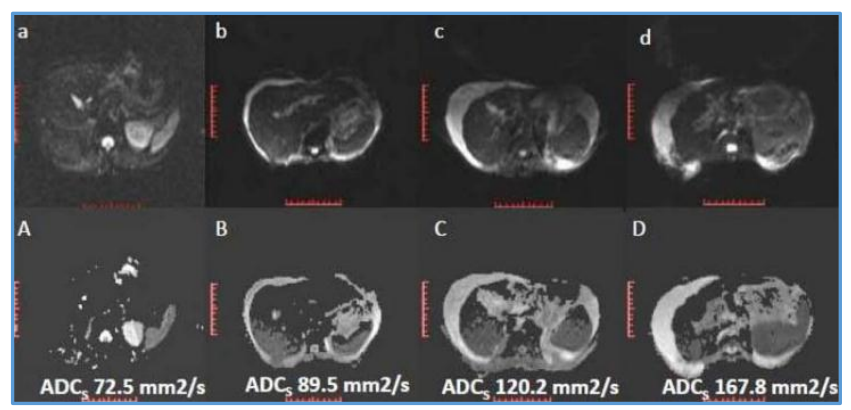

Figure 1. DWI and ADC Mapping of a Normal Study $(a, A)$, Child Class A Cirrhosis (b, B), Child Class B Cirrhosis (c, C) and Child Class C Cirrhosis $(d, D)$ with their respective Spleen $A D C$ Values. ADCs-Spleen ADC

\section{DISCUSSION}

Diffusion weighted imaging has been used extensively in chronic liver disease patients and liver ADC has been found to be a useful adjunct in predicting the degree of fibrosis, especially in patients with viral aetiology. ${ }^{13-16}$ This is due to the reduced perfusion in a fibrotic liver and the degree of ADC reduction implies the severity of fibrosis in the liver. Similarly, cirrhosis is a state of hyperdynamic circulation and in view of portal hypertension there is splanchnic hyperaemia. This splanchnic hyperaemia is assessed in our study by taking spleen as the representative organ of the splanchnic circulation.

In accordance to the recent studies, our initial part of the current study confirmed that liver ADC is significantly reduced in chronic liver disease patients compared with normal subjects. $6,17-22$ This can be attributed to the presence of fibrous tissue, which are collagenous tissue associated with restricted diffusion and subsequent diminished ADC values. However, we also found that spleen ADC is significantly increased in patients with chronic liver disease correlating to their splanchnic hyperaemia. However, this is in contradiction to the earlier reports except the one by Klasen et al, who found negative correlation between spleen and liver ADC in cirrhosis patients. ${ }^{5}$ Hence, in chronic liver disease patients, ADC liver decreases due to liver fibrosis and spleen ADC increases in view of splanchnic hyperaemia.

Normalisation of ADC using a reference organ, which remains relatively constant among patients have been used in previous studies to aid in reduction of ADC calculation variability.6,15,20 Earlier studies used spleen as a representative organ in cirrhotic patients to reduce the ADC calculation variability using ratios. However, the same spleen 
was used for normalisation in studies which correlated liver fibrosis with liver ADC. Our study has proven the negative correlation between the ADC of liver and spleen in chronic liver disease and this suggests that spleen cannot be taken as a reference organ in chronic liver disease patients and this might have confounding influence on the results.

Though earlier studies have used spleen ADC as a reference organ and one recent study correlated spleen ADC with severity of chronic liver disease, our study is the first to correlate spleen ADC to the severity of portal hypertension in chronic liver disease patients. On analysis, we found a significant correlation of the CSPH to the spleen ADC and also to the surrogate markers of portal hypertension like grade of varices and symptomatic hypersplenism. The mechanisms behind the significant positive correlation between spleen ADC and portal hypertension have not been fully elucidated. In theory, elevated portal blood pressure may lead to vasogenic oedema due to sinusoidal congestion and dilatation. As explained earlier there are two mechanisms for portal hypertension in chronic liver disease patients. One is the increased portal venous resistance at the sinusoidal and post sinusoidal level is usually the initiator of portal hypertension. However, increased portal venous inflow secondary to a hyperdynamic systemic circulation and splanchnic hyperaemia is a major contributor to the maintenance of portal hypertension as portosystemic collaterals develop. The cause of the elevated cardiac output and splanchnic hyperaemia is not known, but splanchnic hormones such as glucagon and decreased sensitivity of the splanchnic vasculature to catecholamines probably play a role. Increased production of nitrous oxide and prostacyclin by vascular endothelium is also an important factor. In our study, this splanchnic hyperaemia may be the reason for significant association of ADC value of spleen to CSPH. The high ADC values are consistent with highly mobile water in areas of vasogenic oedema. When we analysed the liver ADC to the severity of portal hypertension, we could not find any significant correlation, implying the heterogeneous correlation of the severity of portal hypertension and severity of chronic liver disease. Similarly, we found that spleen ADC does not correlate with the severity of chronic liver disease (CTP class). These two findings suggest that the spleen ADC is an indirect marker of splanchnic hyperaemia, thereby portal hypertension and liver ADC is an indirect marker for the severity of liver fibrosis. This was also confirmed in our study as well, when we found Liver ADC decreased in cirrhotic patients as evidenced in previous studies and it correlated with CTP class.

One of the greatest hindrance to widespread adoption of DWI in the body is the lack of standardisation. ${ }^{17}$ In prior studies, 23 two different diffusion sequences were used with $b$ values of $0,150,250$ and $400 \mathrm{~mm} 2 / \mathrm{s}$ and 600 and 800 $\mathrm{mm} 2 / \mathrm{s}$, while in another study b values of 0 and $500 \mathrm{~mm} 2 / \mathrm{s}$ were applied. As the $\mathrm{b}$ values increase ADC approaches the true diffusion coefficient, thus minimising the influence of convective motion processes that are sensitive to diffusionhighlighting gradients, mainly perfusion in the randomly organised capillary network. As a general rule lower b values correspond to higher mean ADC, overestimated due to signal contribution from other intravoxel incoherent motions (mainly microvascular perfusion). On the contrary, higher $b$ values lead to lower ADC values as a consequence of the gradient-enhanced signal degradation that eliminates fast diffusion contributions. We took b value of 0,300 and 500 for finding out the true diffusion coefficient.

Though our study had large cohort of patients to study and correlate spleen ADC to the severity of chronic liver disease and portal hypertension than previous studies, we had few limitations in our study. We did not use normalisation of the spleen ADC with a reference organ to have some standardisation to the MRI protocol in view of technical difficulties. Secondly, it was difficult to get patients who were not on medications for portal hypertension. It will be interesting to study the treatment response on the spleen ADC using DWI.

\section{CONCLUSION}

In conclusion, our results show that spleen ADC values vary among patients with liver cirrhosis and control subjects. Furthermore, ADC values in the spleen correlated well with the severity of portal hypertension and not significantly with the degree of liver disease. Thus, the spleen might only be of limited value for normalisation of liver ADC values to determine cirrhosis. ADC measurements may allow for noninvasive evaluation of portal pressure and even in assessment of treatment response.

\section{REFERENCES}

[1] Berzigotti A, Seijo S, Reverter E, et al. Assessing portal hypertension in liver diseases. Expert Rev Gastroenterol Hepatol 2013;7(2):141-55.

[2] Berzigotti A, Castera L. Update on ultrasound imaging of liver fibrosis. J Hepatol 2013;59(1):180-2.

[3] Shin SU, Lee JM, Yu MH, et al. Prediction of esophageal varices in patients with cirrhosis: usefulness of threedimensional MR elastography with echo-planar imaging technique. Radiology 2014;272(1):143-53.

[4] Venkatesh SK, Yin M, Ehman RL. Magnetic resonance elastography of liver: clinical applications. J Comput Assist Tomogr 2013;37(6):887-96.

[5] Klasen J, Lanzman RS, Wittsack HJ, et al. Diffusionweighted imaging (DWI) of the spleen in patients with liver cirrhosis and portal hypertension. Magn Reson Imaging 2013;31(7):1092-6.

[6] Cece H, Ercan A, Yildiz S, et al. The use of DWI to assess spleen and liver quantitative ADC changes in the detection of liver fibrosis stages in chronic viral hepatitis. Eur J Radiol 2013;82(8):e307-12.

[7] Kele PG, van der Jagt EJ. Diffusion weighted imaging in the liver. World J Gastroenterol 2010;16(13):1567-76.

[8] Hong Y, Shi Y, Liao W, et al. Relative ADC measurement for liver fibrosis diagnosis in chronic hepatitis B using spleen/renal cortex as the reference organs at $3 \mathrm{~T}$. Clin Radiol 2014;69(6):581-8.

[9] Malinchoc M, Kamath PS, Gordon FD, et al. A model to predict poor survival in patients undergoing transjugular intrahepatic portosystemic shunts. Hepatology 2000;31(4):864-71.

[10] Conn HO. Ammonia tolerance in the diagnosis of esophageal varices. A comparison of endoscopic, radiologic, and biochemical techniques. J Lab Clin Med 1967;70(3):442-51. 
[11] Rajalingam R, Javed A, Sharma D, et al. Management of hypersplenism in non-cirrhotic portal hypertension: a surgical series. Hepatobiliary Pancreat Dis Int 2012;11(2):165-71.

[12] Ong JP, Aggarwal A, Krieger D, et al. Correlation between ammonia levels and the severity of hepatic encephalopathy. Am J Med 2003;114(3):188-93.

[13] Trifan A, Cojocariu C, Sfarti C, et al. Non-invasive evaluation of liver fibrosis in chronic hepatitis C. Rev Med Chir Soc Med Nat Iasi 2012;116(1):135-8.

[14] Denzer UW, Luth S. Non-invasive diagnosis and monitoring of liver fibrosis and cirrhosis. Best Pract Res Clin Gastroenterol 2009;23(3):453-60.

[15] Do RK, Chandarana H, Felker E, et al. Diagnosis of liver fibrosis and cirrhosis with diffusion-weighted imaging: value of normalized apparent diffusion coefficient using the spleen as reference organ. AJR Am J Roentgenol 2010;195(3):671-6.

[16] Enomoto M, Morikawa H, Tamori A, et al. Noninvasive assessment of liver fibrosis in patients with chronic hepatitis B. World J Gastroenterol 2014;20(34):12031-8.

[17] Girometti R, Furlan A, Esposito G, et al. Relevance of bvalues in evaluating liver fibrosis: a study in healthy and cirrhotic subjects using two single-shot spin-echo echo-planar diffusion-weighted sequences. J Magn Reson Imaging 2008;28(2):411-9.
[18] Sandrasegaran K, Tahir B, Patel A, et al. The usefulness of diffusion-weighted imaging in the characterization of liver lesions in patients with cirrhosis. Clin Radiol 2013;68(7):708-15.

[19] Soylu A, Kilickesmez O, Poturoglu S, et al. Utility of diffusion-weighted MRI for assessing liver fibrosis in patients with chronic active hepatitis. Diagn Interv Radiol 2010;16(3):204-8.

[20] Tokgöz O, Unal I, Turgut GG, et al. The value of liver and spleen ADC measurements in the diagnosis and follow up of hepatic fibrosis in chronic liver disease. Acta Clin Belg 2014;69(6):426-32.

[21] Hu XR, Cui XN, Hu QT, et al. Value of MR diffusion imaging in hepatic fibrosis and its correlations with serum indices. World J Gastroenterol 2014;20(24):7964-70.

[22] Sugimoto R, Iwasa M, Maeda M, et al. Value of the apparent diffusion coefficient for quantification of low-grade hepatic encephalopathy. Am J Gastroenterol 2008;103(6):1413-20.

[23] Koh DM, Collins DJ. Diffusion-weighted MRI in the body: applications and challenges in oncology. AJR Am J Roentgenol 2007;188(6):1622-35. 